\title{
NEW APPROACH TO THE DETECTION OF EPILEPTIFORM ACTIVITY IN EEG SIGNALS AND METHODS TO DIFFERENTIATE EPILEPTIC SEIZURES FROM CHEWING ARTIFACTS
}

\section{Ivan A. Kershner, Yuri V. Obukhov}

Kotelnikov Institute of Radioengineering and Electronics of RAS, http://www.cplire.ru/

11/7, Mokhovaya str., Moscow 125009, Russian Federation

\section{Mikhail V. Sinkin}

Sklifosovsky Scientific Research Institute for Emergency Medicine of Moscow Department of Health, https://sklif.mos.ru/

3, Sukharevskaya sq., Moscow 129090, Russian Federation

ivan_kershner@mail.ru,mvsinkin@gmail.com, yuvobukhov@mail.ru

Abstract. The new approach based on Morlet wavelet spectrograms ridges analysis and allowing automatic detecting different activity in long term EEG signals is developed. To distinguish epileptiform activity from chewing artifacts two approaches are proposed. The quantitative characteristics of events wavelet spectrogram ridges were studied, as well as the frequency of broadband peaks at time points corresponding to peak-wave epileptiform activity on the one hand, and the peaks of myographic activity during chewing on the other hand. Signs by which one can qualitatively divide the group containing epileptic discharges from chewing artifacts were found. Keywords: EEG; wavelet spectrogram; ridges; epileptic seizure; chewing artifacts; seizure detection UDC 004.67, 004.93"'11

Bibliography - 8 references

Received 28.06.2019, accepted 2.07.2019

RENSIT, 2019, 11(2):237-242

DOI: 10.17725/rensit.2019.11.237

CONTENTS

1. INTRODUCTION (237)

2. Approach (238)

3. Signal PROCESSING (238)

4. Conclusion (241)

REFERENCES (241)

\section{INTRODUCTION}

During data analysis of long-term electroencephalographic (EEG) of patients with traumatic brain injury neurophysiologists manually mark up the EEG records by comparing individual fragments of EEG with epileptic seizures, chewing artifacts, etc. That is time-consuming process when marking daily and longer EEG signals. Automatic marking of epileptic and epileptiform seizures in EEG signals of patients with posttraumatic epilepsy, as well as differentiation seizures fragments from chewing artifacts will help to neurophysiologists accelerate the process of detecting epileptiform seizures.

The problem of detecting epileptic seizures of various types [1], as well as various artifacts [2] is widely known and remains relevant. For example, in [3] attention is paid to the finding of muscle artifacts, including chewing artifacts through the study of amplitude and frequency components. In paper proposes to divide the signal into epochs and calculate the minimum, maximum amplitude values and the first derivative, the absolute and relative powers of the Fourier spectrum, the boundary frequency of the spectrum, up to which the sum of the spectral power is at the level of $95 \%$ of the total spectral power of the entire spectrum. Then the parameters were compared with the parameters of the control record, which does not contain artifacts. Control record is selected manually for each EEG record. It should be noted that the method is aimed at finding artifacts with a frequency of more than $25 \mathrm{~Hz}$.

The article [4] presents the use of support vector machine (SVM) to distinguish different types of artifacts. The features for SVM are obtained from EEG and gyroscopic channels. 
Records are collected from 7 people who do not have any neurological and psychiatric disorders. The subject had to make five kinds of movements similar to motion artifacts. Each action records within 30 seconds, then 20 seconds of a break as a control record without motion artifacts. Automatic detection of artifacts by the method is not provided.

The paper [5] describes application of Bayes statistics to real-time recognition of epileptic seizures in with the removal of artifacts associated with wire breakage and muscle activity outside the range of 12-25 Hz. Alpha activity (8-13 Hz), characteristic of both artifacts and epileptic seizures $(3-25 \mathrm{~Hz})$, is marked as seizure, i.e. differentiation between epileptic seizure and artifacts in this frequency range was not carried out.

The article [6] presents the method of recognition of epileptic seizures based on Fourier analysis. The method works fast enough that it can be used in the system of informing doctors about the onset of the seizure, but at the same time, the authors provide information that the analysis of EEG signals by their method did not recognize epileptic seizures in EEG of 3 patients. Neurophysiology experts at the same time mark 3, 5 and 6 epileptic seizures for them.

We propose a new approach based on Morlet wavelet spectrograms ridges analysis for the automatic detection of all suspicious fragments of electroencephalographic signals of patients with posttraumatic epilepsy on long term EEG records (a day or more) with the possibility of their further classification.

\section{APPROACH}

Since EEG signals contain both artifacts and epileptic discharges, there is a need for their automatic detection. It is proposed to find the beginning and the end of signal fragments in which suspicious activity with increased values of the power spectral density is observed by segmenting the Morlet wavelet spectrogram ridges. For that purpose, we calculate the histogram of the ridges of the wavelet spectrograms. For fragmented ridges, frequency and time parameters are calculated, according to which it is possible to classify the fragments of the ridge into the corresponding event in the future. In addition, sections of wavelet spectrograms in frequency are considered to identify additional features of the difference between epileptic seizures and chewing artifacts.

\section{SIGNAL PROCESSING}

Consider the approach to detect specific events step by step on the example of a signal containing an epileptic seizure, obtained in the Department of neurosurgery research of Sklifosovsky Scientific Research Institute for Emergency Medicine (Fig. 1).

Initially, the signal is filtered by 2 nd order Butterworth bandpass filter with a bandwidth of 0.5 to $22 \mathrm{~Hz}$, as well as notch filters multiples of $25 \mathrm{~Hz}$ to remove noise and network interference. Then the wavelet spectrogram $S$ is calculated as follows [7]:

$$
\begin{aligned}
& S(\tau, f)=|W(\tau, f)|^{2}, \\
& W(\tau, f)=\sqrt{f} \int x(t) \psi^{*}((t-\tau) f) d t \\
& \psi(\eta)=\frac{1}{\sqrt{\pi F_{b}}} \exp \left(2 \pi i F_{c} \eta\right) \exp \left(\frac{\eta^{2}}{F_{b}}\right),
\end{aligned}
$$

where $\psi^{*}$ is the complex conjugation of the Morlet mother function $\psi, F_{c}=F_{b}=1, x$ is the original signal, and $W$ is the wavelet transform.

At each $t_{\mathrm{i}}$ time moment in the frequencypower spectral density (PSD) plane, the maximum values of the PSD are calculated. Then the phase characteristic $P$ of the signal $x$ is calculated:

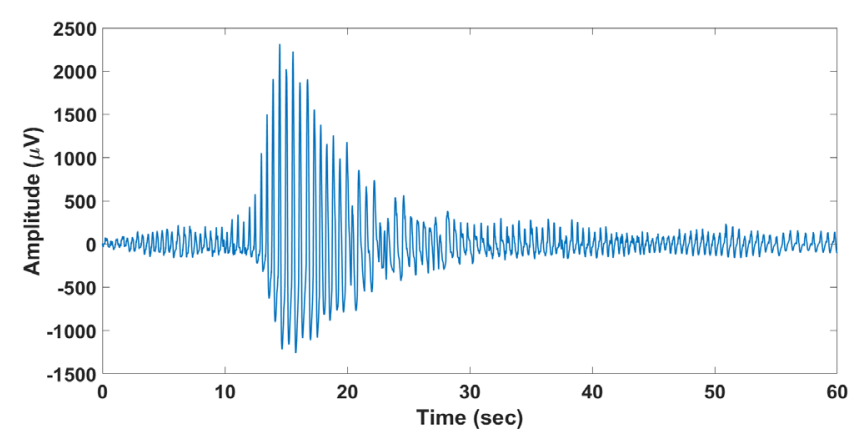

Fig. 1. The signal of EEG containing an epileptic seizure. 


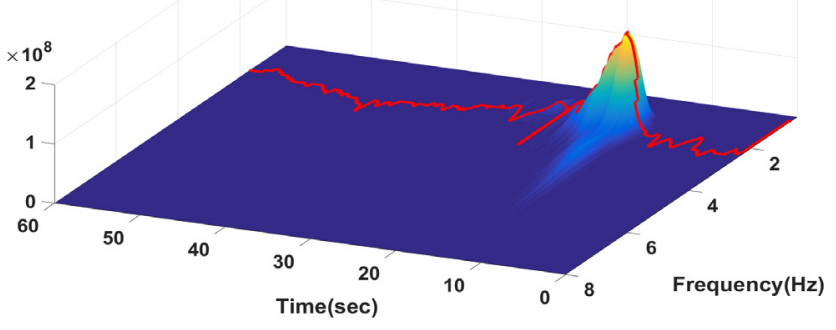

Fig. 2. Wavelet spectrogram of an EEG signal containing an epileptic seizure, projected on the frequency-time-PSD axis with maximum PSD values (red line).

$$
P(k)=\tau_{k} f_{r}\left(\tau_{k}\right),
$$

where $f_{\mathrm{r}}\left(\tau_{\mathrm{k}}\right)$ is the instantaneous frequency of the signal at the time $\tau_{\mathrm{k}}$. Moreover, $f_{\mathrm{r}}\left(\tau_{\mathrm{k}}\right)$ coincides with the frequency value at the point of the wavelet spectrogram ridge at the time $\tau_{k}$, if the following condition is satisfied [8]:

$$
\frac{\tau^{2} F_{b}}{2} \frac{d^{2} P}{d \tau^{2}}<<1
$$

Further, the ridge points that do not satisfy the inequality (5) wavelet spectrograms should be removed from the consideration. The line connecting points with the maximum values of PSD on the wavelet spectrogram, which have the property of phase stationarity (5), is called the ridge of the wavelet spectrogram. Fig. 2 shows a wavelet spectrogram in the frequencytime-power spectral density projection with a calculated ridge. It shows a peak in the time period from 10 to 30 seconds, which neurophysiologists refer to an epileptic seizure.

Further, the histogram of the number of fragments depending on the selected PSD

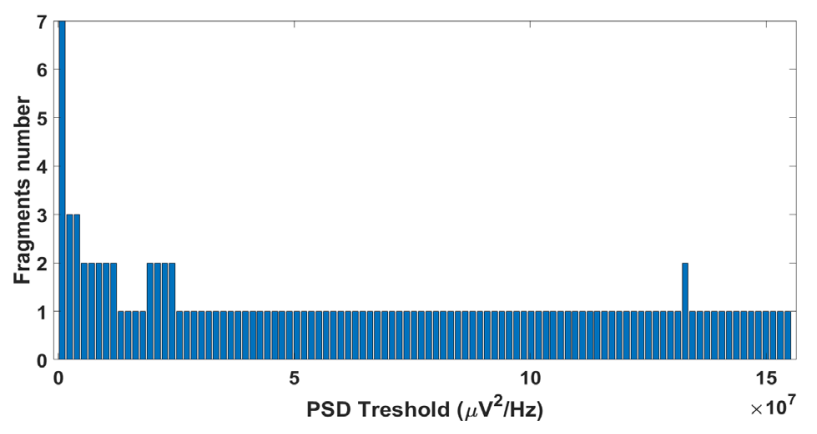

Fig. 3. Histogram of fragments of the ridge of the wavelet spectrogram for the threshold values of spectral power density.

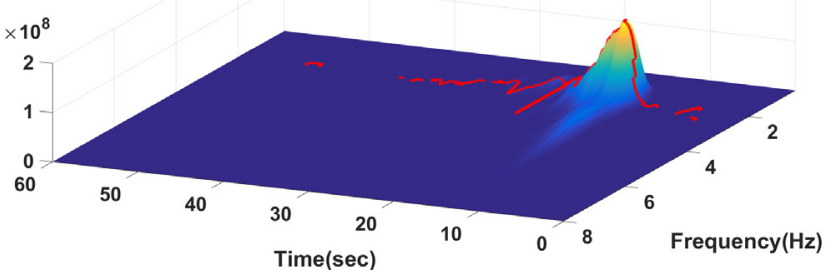

Fig. 4. A fragmented ridge of the wavelet spectrogram of an EEG signal containing an epileptic seizure, with remote points in accordance with the formula (5).

threshold value is analyzed to separate the background and activity (Fig. 3). The threshold at which the maximum number of ridge fragments is selected. At Fig. 3 PSD $D_{\text {threshold }}=1.2 \cdot 10^{7} \mu \mathrm{V}^{2} /$ Hz. Fragments of the ridge, in this case, are such points of the ridge, which PSD values are above the threshold and are next to each other in sequence number. An example of a fragmented ridge is shown in Fig. 4.

The following are calculated for each fragment: duration; maximum and minimum values of frequency and spectral power density; time at which the maximum value of the spectral power density was recorded; arithmetic mean and standard deviation of frequency.

Table 1 shows the values of the calculated parameters for EEG records containing epileptic seizures and chewing artifacts.

From the data given in the table, the maximum and minimum frequency values and the mean frequency in chewing artifacts are lower than in

Table 1

Parameters calculated for epileptic seizures (ES) and chewing artifacts (CA).

\begin{tabular}{|l|l|l|l|l|l|l|l|l|l|}
\hline $\begin{array}{l}\text { Acti- } \\
\text { vity }\end{array}$ & $T_{\mathrm{b}}-T_{\mathrm{e}}$ & $F_{\min }$ & $F_{\max }$ & $F_{\text {std }}$ & $F_{\operatorname{man}}$ & $\begin{array}{l}F_{\text {sdd }} \\
F_{\text {man }}\end{array}$ & $P S D_{\max }$ & $P S D_{\min }$ & $T_{\text {Pmax }}$ \\
\hline CA & $100.5-103.8$ & 0.50 & 0.50 & 0.00 & 0.50 & 0.00 & $5.08 \mathrm{E}+06$ & $1.03 \mathrm{E}+06$ & 102.42 \\
\hline CA & $198-201$ & 0.70 & 1.00 & 0.07 & 0.87 & 0.08 & $1.38 \mathrm{E}+06$ & $2.37 \mathrm{E}+05$ & 199.21 \\
\hline CA & $270.2-273.9$ & 0.50 & 0.50 & 0.00 & 0.50 & 0.00 & $9.95 \mathrm{E}+06$ & $2.10 \mathrm{E}+06$ & 272.09 \\
\hline CA & $3-9.39$ & 0.50 & 0.70 & 0.06 & 0.62 & 0.10 & $2.65 \mathrm{E}+05$ & $6.05 \mathrm{E}+04$ & 7.83 \\
\hline CA & $16.49-22.49$ & 0.50 & 0.50 & 0.00 & 0.50 & 0.00 & $3.98 \mathrm{E}+05$ & $7.83 \mathrm{E}+04$ & 19.30 \\
\hline CA & $26.29-30.09$ & 0.50 & 0.70 & 0.08 & 0.64 & 0.12 & $3.53 \mathrm{E}+05$ & $6.01 \mathrm{E}+04$ & 28.38 \\
\hline ES & $133.68-139.9$ & 1.60 & 1.90 & 0.08 & 1.79 & 0.04 & $1.43 \mathrm{E}+08$ & $3.28 \mathrm{E}+07$ & 135.44 \\
\hline ES & $85.5-92.1$ & 2.80 & 6.20 & 0.56 & 4.09 & 0.14 & $7.27 \mathrm{E}+06$ & $4.85 \mathrm{E}+05$ & 90.65 \\
\hline ES & $92.41-98$ & 2.10 & 3.60 & 0.32 & 3.08 & 0.10 & $4.31 \mathrm{E}+06$ & $3.23 \mathrm{E}+05$ & 93.22 \\
\hline
\end{tabular}

$T_{\mathrm{b}}$ and $T_{\mathrm{e}}$ are the start and end time of activity; $F_{\text {min }}$ and $F_{\text {max }}$ - minimum and maximum frequency value; $F_{\text {mean }}$ and $F_{\text {std }}$ is the mean and the standard deviation; $P S D_{\max }$ and $P S D_{\min }^{\text {std }}-$ minimum and maximum values of $P S D ; T_{P \max }^{\max }-$ time, when there was higher $P S D$ value. 
Parameters calculated for epileptic seizures.

\begin{tabular}{|l|l|l|l|l|l|l|l|l|}
\hline$T_{\mathrm{b}}-T_{\mathrm{e}}$ & $F_{\text {min }}$ & $F_{\text {max }}$ & $F_{\text {std }}$ & $F_{\text {mean }}$ & $\begin{array}{l}F_{\text {std }} \\
F_{\text {mean }}\end{array}$ & $P S D_{\text {max }}$ & $P S D_{\text {min }}$ & $T_{\text {Pmax }}$ \\
\hline $1.99-7.99$ & 0.60 & 0.80 & 0.07 & 0.67 & 0.10 & $3.02 \mathrm{E}+04$ & $9.63 \mathrm{E}+03$ & 6.78 \\
\hline $122.49-124.49$ & 0.90 & 1.60 & 0.21 & 1.21 & 0.17 & $3.67 \mathrm{E}+04$ & $1.13 \mathrm{E}+04$ & 123.55 \\
\hline $128.2-129.39$ & 1.70 & 2.00 & 0.09 & 1.78 & 0.05 & $3.25 \mathrm{E}+04$ & $1.11 \mathrm{E}+04$ & 128.77 \\
\hline $356.49-358.79$ & 0.70 & 1.10 & 0.13 & 0.96 & 0.14 & $2.40 \mathrm{E}+04$ & $7.90 \mathrm{E}+03$ & 357.77 \\
\hline $83.7-85.29$ & 1.20 & 2.40 & 0.42 & 1.82 & 0.23 & $1.78 \mathrm{E}+05$ & $3.06 \mathrm{E}+04$ & 84.73 \\
\hline $98.7-98.39$ & 3.90 & 4.40 & 0.14 & 4.08 & 0.03 & $2.32 \mathrm{E}+05$ & $9.22 \mathrm{E}+04$ & 99.03 \\
\hline $100-100.39$ & 3.20 & 3.80 & 0.20 & 3.45 & 0.06 & $1.79 \mathrm{E}+05$ & $9.04 \mathrm{E}+04$ & 100.27 \\
\hline $100.79-101.19$ & 3.50 & 4.30 & 0.19 & 3.92 & 0.05 & $1.36 \mathrm{E}+05$ & $6.59 \mathrm{E}+04$ & 101.03 \\
\hline $102-102.69$ & 2.90 & 3.30 & 0.09 & 3.08 & 0.03 & $2.93 \mathrm{E}+05$ & $9.11 \mathrm{E}+04$ & 102.35 \\
\hline $103.79-104.29$ & 3.30 & 4.90 & 0.53 & 3.77 & 0.14 & $2.82 \mathrm{E}+05$ & $9.90 \mathrm{E}+04$ & 104.09 \\
\hline $104,79-105,29$ & 2.20 & 2.80 & 0.14 & 2.55 & 0.05 & $1.61 \mathrm{E}+05$ & $1.01 \mathrm{E}+05$ & 105.02 \\
\hline $106,09-106,49$ & 2.90 & 5.30 & 0.99 & 3.99 & 0.25 & $1.29 \mathrm{E}+05$ & $8.47 \mathrm{E}+04$ & 106.20 \\
\hline $113,59-118,09$ & 1.70 & 2.40 & 0.12 & 2.15 & 0.06 & $2.41 \mathrm{E}+05$ & $3.83 \mathrm{E}+04$ & 115.75 \\
\hline
\end{tabular}

epileptic discharges.

If we take $F_{\text {min }}=0.7, F_{\text {max }}=1, F_{\text {mean }}=0.87$ as the threshold values, then the two groups are well separated. Consider the parameters of epileptic discharges only (Table 2).

At selected thresholds $F_{\text {min }}=0.7, F_{\text {max }}=1$, $F_{\text {mean }}=0.87$, only one epileptic seizure from table 2 has characteristics similar with chewing artifacts parameters. In the remaining 12 cases, epileptic seizures satisfy the specified conditions.

Another approach to differentiate epileptic activity from chewing artifacts is to analyze the broadband peaks of wavelet. The peaks of the EEG signal during the seizure and chewing are sharp, so the wavelet spectrograms at the time of these peaks are broadband. Therefore, in addition to calculating the parameters of the

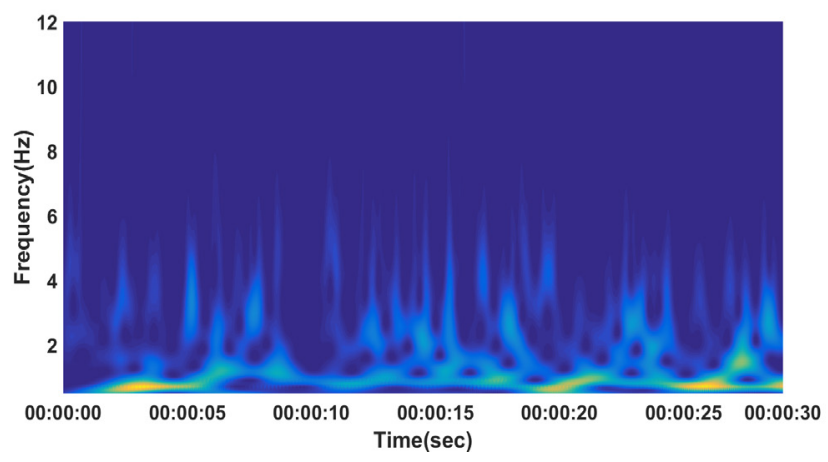

Fig. 5. Wavelet spectrogram of an EEG signal containing a chewing artifact.

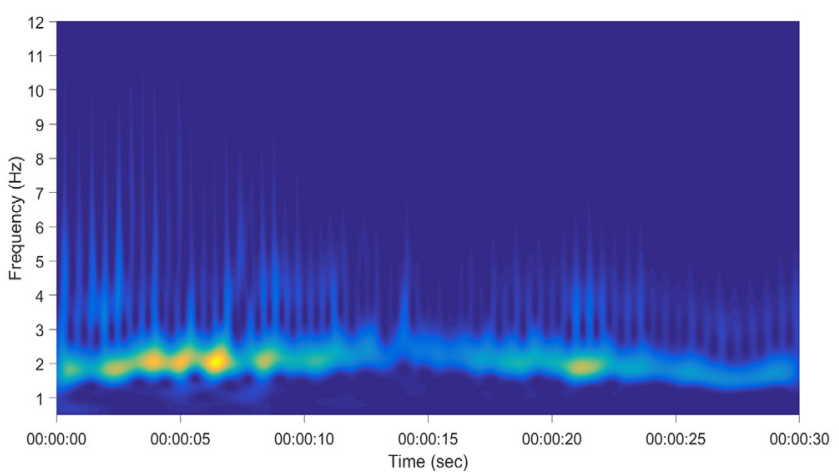

Fig. 6. Wavelet spectrogram of an EEG signal containing an epileptic seizure.

ridges, the frequency of broadband peaks of EEG wavelet spectrograms was studied.

We describe the new approach by the example of fragments of EEG wavelet spectrograms with a chewing artifact and epileptic seizure presented in Fig. 5 and 6. From the matrix $S$ (1) were selected the values in the wavelet rows corresponding to the frequency $F_{\text {cur }}$ from $3.5 \mathrm{~Hz}$ to $6 \mathrm{~Hz}$ with step $0.5 \mathrm{~Hz}$ over the entire time interval:

$$
V_{c u r}\left(\tau_{k}\right)=S\left(\tau_{k}, F_{c u r}\right), \tau_{k}=0: T \text {. }
$$

Vector $V_{\text {cur }}$ represents a raw of the wavelet spectrogram at the frequency $F_{\text {cur. }}$. For the resulting vectors $V_{\text {cur }}$ calculated Fourier spectra. Fig. 7 shows examples of Fourier spectra of $V_{\text {cur }}$ for epileptic seizure and chewing artifact at $F_{\text {cur }}=4 \mathrm{~Hz}$ on wavelet spectrogram. For each Fourier spectrum, the frequency at which the main peak and half-width of the main peak are located at half-height for each frequency level of the wavelet spectrogram was calculated.

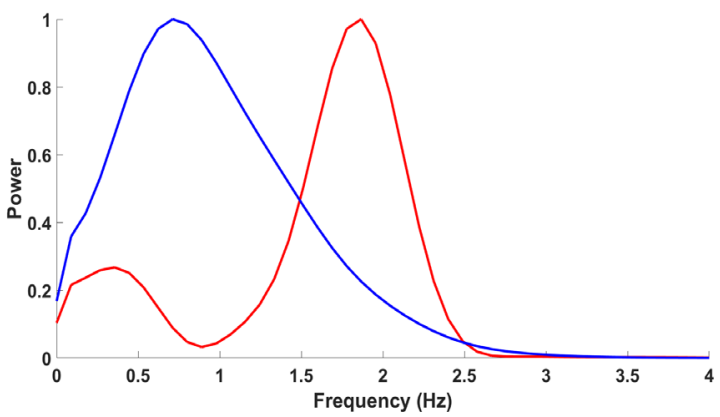

Fig. 7. Fourier spectra at the frequency of the wavelet spectrogram EEG Fcur $=4 \mathrm{~Hz}$. The red line is the Fourier Spectrum for an epileptic seizure, the blue line for a chewing artifact. 
Table 3

The parameters of the Fourier spectra of the wavelet spectrograms rows of the EEG with chewing and epileptic seizure.

\begin{tabular}{|c|c|c|c|c|}
\hline $\begin{array}{c}\text { Wavelet } \\
\text { spectro- } \\
\text { gram } \\
\text { frequency, } \\
\mathrm{Hz}\end{array}$ & $\begin{array}{c}\text { Peak } \\
\text { frequency } \\
\text { (chewing) }\end{array}$ & $\begin{array}{c}\text { Peak } \\
\text { frequency } \\
\text { (epileptic } \\
\text { seizure) }\end{array}$ & $\begin{array}{c}\text { Half- } \\
\text { width } \\
\text { of peak } \\
\text { (chewing) }\end{array}$ & $\begin{array}{c}\text { Half- } \\
\text { width } \\
\text { of peak } \\
\text { (epileptic } \\
\text { seizure) }\end{array}$ \\
\hline 3.5 & 0.71 & 1.86 & 1.06 & 0.62 \\
\hline 4 & 0.71 & 1.86 & 1.2 & 0.64 \\
\hline 4.5 & 0.71 & 1.86 & 1.25 & 0.69 \\
\hline 5 & 0.71 & 1.86 & 1.33 & 0.65 \\
\hline 5.5 & 0.71 & 1.95 & 1.51 & 0.67 \\
\hline 6 & 0.71 & 1.95 & 1.91 & 0.63 \\
\hline
\end{tabular}

Table 3 shows the frequency at which the main peak and the half-width of the main peak are located at half-height, depending on the wavelet spectrogram frequency.

Fig. $7 F_{\text {cur }}=4 \mathrm{~Hz}$ shows differences between epileptic seizures and chewing artifacts in the frequency of the Fourier spectrum peak, for example, for the chewing artifact it is at $0.71 \mathrm{~Hz}$, and for the epileptic seizure at $1.86 \mathrm{~Hz}$. This can be interpreted as the presence of peak-wave activity in an epileptic seizure. The half-width of the Fourier spectrum peaks in the chewing artifact is almost 2 times greater than that of an epileptic seizure. This may mean greater stability of the peak-wave activity period during the seizure compared to chewing. With an increase in the frequency of wavelet spectrograms of EEG signals, the frequency ratio of the Fourier peaks of the epileptic seizure to chewing remains approximately the same. While the half-width of the Fourier spectrum peaks increases for chewing artifacts.

\section{CONCLUSION}

A new approach for detection epileptic seizures and chewing artifacts in electroencephalographic signals based on the analysis of their wavelet spectrograms and finding the parameters of wavelet spectrogram ridges is presented. It was found that the maximum value of the frequency and the standard deviation of the frequency of the wavelet spectrogram ridge can be attributed to the epileptic discharge or chewing artifact. We studied the wavelet spectrogram rows at frequencies larger than ridge frequency. It is shown that on Fourier spectra of wavelet spectrogram rows at frequencies from 3.5 to 6 $\mathrm{Hz}$ at epileptic discharge, the frequency of the peak of the wavelet spectrogram rows is more than 2.5 times higher than the frequency of the Fourier peak of the wavelet spectrogram for chewing. The half-width of the Fourier picks of rows of the wavelet spectrograms of the EEG on the frequencies above $3.5 \mathrm{~Hz}$ at chewing artifacts in 1.5-3 times more compared to the half-width of the Fourier spectra in epileptic seizure. This meaning greater stability of the peak-wave activity period during the seizure compared to chewing. By combining those two approaches it is possible to reliably differentiate an epileptic seizure from a chewing artifact.

\section{ACKNOWLedgments}

The work was carried out within the framework of the state task and partially was supported by the Russian Foundation for Basic Research, the project No 18-2902035.

\section{REFERENCES}

1. Fergus P, Hignett D, Hussain A, Al-Jumeily D, Abdel-Aziz K. Automatic epileptic seizure detection using scalp EEG and advanced artificial intelligence techniques. BioMed research international, 2015, vol. 2015, article ID 986736, 17 pages; doi: 10.1155/2015/986736.

2. Islam MK, Rastegarnia A, Yang Z. Methods for artifact detection and removal from scalp EEG: A review. Neurophysiologie Cliniquel Clinical Neurophysiology, 2016, 46(4-5):287305.

3. Van de Velde M, van Erp G, Cluitmans PJ. Detection of muscle artefact in the normal human awake EEG. Electroencephalography and clinical Neurophysiology, 1998, 107(2):149-158.

4. O'Regan S, Faul S, Marnane W. Automatic detection of EEG artefacts arising from head movements using EEG and gyroscope signals. Medical engineering \& physics, 2013, 
35(7):867-874.

5. Saab ME, Gotman J. A system to detect the onset of epileptic seizures in scalp EEG. Clinical Neurophysiology, 2005, 116(2):427-442.

6. Hopfengärtner R, Kasper B, Graf W, Gollwitzer S, Kreiselmeyer G, Stefan H, Hamer $\mathrm{H}$. Automatic seizure detection in long-term scalp EEG using an adaptive thresholding technique: a validation study for clinical routine. Clinical Neurophysiology, 2014, 125(7):1346-1352.

7. Mallat SG. $A$ Wavelet Tour of Signal Processing. The Sparse Way. Burlington, Academic Press, 2009, $562 \mathrm{p}$.

8. Tolmacheva RA, Obukhov YuV, Polupanov AF, Zhavoronkova LA. New Approach to Estimation of Interchannel Phase Coupling of Electroencephalograms. Journal of Communications Technology and Electronics, 2018, 63(9):1070-1075. 